\title{
WAVE SPEEDS FOR AN ELASTOPLASTIC MODEL FOR TWO-DIMENSIONAL DEFORMATIONS WITH A NONASSOCIATIVE FLOW RULE
}

\author{
BY \\ MICHAEL GORDON (Dept. of Mathematics, North Carolina State University, Raleigh, NC) \\ AND \\ F. XABIER GARAIZAR (Dept. of Mathematics, North Carolina State University, Raleigh, NC)
}

Summary. A system of partial differential equations describing elastoplastic deformations in two space dimensions is studied. The constitutive relations for plastic deformation include a nonassociative flow rule and shear strain hardening. After a change of variables, the characteristic speeds of plane wave solutions of the system are computed. For both plastic and elastic deformations, there are two nonzero wave speeds, referred to as fast and slow waves. It is shown that there are regions in stress space for which the speed of fast plastic waves exceeds the speed of fast elastic waves, which translates into a lack of uniqueness for certain initial value problems and introduces nontrivial difficulties for numerical methods. Finally, these regions are computed for an example using representative constitutive data.

1. Introduction. In this paper we study a system of partial differential equations describing granular flow in two space dimensions. The equations under consideration, studied by Schaeffer [11] among others, include a nonassociative flow rule. Flow rules that are nonassociative are common to a range of models describing the deformation of geological and granular materials (cf. [3], [4], [8]). They are supported by extensive experimental data; see, for example, Vardoulakis and Graf [14].

Sandler and Rubin [10] observed that for planar waves, nonassociativity in dynamical problems may imply that, at certain values of stress, elastic waves would travel slower than plastic waves. They showed that this order of the elastic and plastic waves translates into a lack of uniqueness for initial value problems and argued that it would introduce nontrivial difficulties for numerical methods. Schaeffer and Shearer [12], in the context of scale-invariant problems, showed that uniqueness of the initial value problem can be reestablished when an appropriate entropy condition is introduced. In this case, problems may arise with the existence of solutions.

Received August 1, 1996.

1991 Mathematics Subject Classification. Primary 73E05, 73E70, 73D99, 35L67.

The second author was supported by NSF Grant DMS 9201115, which includes funds from AFOSR. 
We study planar waves associated to the system of partial differential equations as derived in [11]. Depending on the level of loading, the deformation described by the system will be elastic or plastic. In each case, the wave structure of the system consists of a pair of stationary waves and two other families of waves: fast and slow. The main result of this paper is Theorem 5, where we show that there is a region in stress space for which the elastic fast waves travel slower than the plastic fast waves. This result relates to that of Sandler and Rubin [10], although our emphasis is different. If the plastic waves travel faster than the elastic waves, initial value problems with Riemann initial data (Riemann problems) will lack self-similar solutions that exclude shock waves. In general, an elastic state will connect to a plastic state with a wave curve consisting of a loading elastic wave, placing the stress on the yield curve, followed by a plastic rarefaction wave. If the state behind the elastic wave (with stress on the yield curve) has values of stress such that the plastic wave speed is larger than the elastic wave speed, such a connection is not possible. Indeed the presence of impinging characteristics is the classical introduction of shock waves (plastic shocks for this case).

In the context of our problem, plastic shocks were excluded since it is unclear how to incorporate shock waves into the present model. The system is not in conservation form and it is necessary to define an associated viscous system (see [5], [6]) in order to obtain a proper characterization of the shock waves. In the present model, there is no natural formulation of such a viscous system. Many numerical methods rely on the resolution of Riemann Problems. Therefore the lack of solutions for Riemann Problems implies that those numerical methods could fail during simulations where the stress is in the region described by Theorem 5 and the plastic waves travel faster than the elastic waves.

The system derived in [11] was simplified by ignoring the rotational terms of the Jaumann stress rate. It is pointed out there that these terms are small when the mean and shear stresses $\tau$ and $\sigma$ are small in comparison to the shear modulus $G$, as they are for the representative constitutive data considered in [11]. For the sake of completeness, Section 5 of this work considers the effect of retaining the rotational terms. We will show (Theorem 9) that keeping the rotational terms in the system does not eliminate the reverse ordering of the waves and the qualitative behavior of the wave speeds remains the same. We complete this paper with an example in which, for some representative constitutive data, we calculate the regions where the reverse ordering of the wave speeds occurs. We show that the size and location of these regions makes them relevant to the model and hence they cannot be ignored.

We conclude from this work that although nonassociative models are valuable for the description of slow granular flow, and clearly suggested by the experimental data, one should at least be very cautious with their use. A deeper understanding of the role of the reverse ordering of the fast wave speeds is needed. This could involve the inclusion of entropy conditions to establish uniqueness of initial value problems as in [12], addition of plastic shock waves or a modification of the model itself, in order to define plastic shock waves as weak solutions or eliminate altogether the reverse ordering of the waves. As we remarked above, if plastic waves were to be included, since the system is not in conservation form, a related viscous system would be needed. 
2. The model equations. First, we briefly review the two-dimensional granular flow model derived in [11]. The fundamental variables are the density $\rho$, the velocity $\overrightarrow{\mathbf{v}}=\left(v_{1}, v_{2}\right)^{\mathrm{T}}$, and the Cauchy stress tensor

$$
\mathbf{T}=\left[\begin{array}{ll}
T_{11} & T_{12} \\
T_{12} & T_{22}
\end{array}\right],
$$

which we assume has positive eigenvalues $\sigma_{1} \geq \sigma_{2}>0$ (corresponding to compressive stresses). These variables are subject to equations expressing the conservation of mass and momentum,

$$
\begin{array}{r}
\partial_{t} \rho+\rho \nabla \cdot \overrightarrow{\mathbf{v}}=0, \\
\rho \partial_{t} \overrightarrow{\mathbf{v}}+\nabla \cdot \mathbf{T}=0,
\end{array}
$$

where $\nabla=\left(\partial_{x}, \partial_{y}\right)^{\mathrm{T}}$. (We omit the convective terms $\overrightarrow{\mathbf{v}} \cdot \nabla$ in the material derivative, as these would only mean a translation of the eigenvalues of the system in consideration, and thus would not affect our results. Also, since the model describes slow flow, omission of these terms is reasonable.)

The strain rate tensor $\mathbf{V}$ is expressed in terms of the velocity as

$$
\mathbf{V}=\frac{1}{2}\left(\nabla \overrightarrow{\mathbf{v}}^{\mathrm{T}}+\left(\nabla \overrightarrow{\mathbf{v}}^{\mathrm{T}}\right)^{\mathrm{T}}\right) .
$$

We decompose $\mathbf{V}$ into its elastic and plastic parts,

$$
\mathbf{V}=\mathbf{V}^{(\mathrm{e})}+\mathbf{V}^{(\mathrm{p})}
$$

and assume that the elastic strain rate satisfies the constitutive relation of linear elasticity,

$$
\frac{1}{2 G}\left(\partial_{t} \mathbf{T}-\nu\left(\operatorname{Tr} \partial_{t} \mathbf{T}\right) \mathbf{I}\right)+\mathbf{V}^{(\mathrm{e})}=0
$$

where $G$ is the shear modulus, $\nu$ is Poisson's ratio, and Tr denotes the trace of a tensor,

$$
\operatorname{Tr} \mathbf{A}=A_{11}+A_{22} .
$$

The plastic strain rate satisfies the constitutive relation (known as the flow rule)

$$
\mu \Psi(\mathbf{T})+\mathbf{V}^{(\mathbf{p})}=0
$$

where $\mu$ is a nonnegative scalar variable and

$$
\Psi(\mathbf{T})=\sqrt{2} \frac{\operatorname{dev} \mathbf{T}}{|\operatorname{dev} \mathbf{T}|}-\beta \mathbf{I}, \quad|\beta|<1,
$$

where $\operatorname{dev} \mathbf{T}=\mathbf{T}-\frac{1}{2}(\operatorname{Tr} \mathbf{T}) \mathbf{I}$ denotes the deviator of the stress tensor $\mathbf{T}$. Here $\beta$, a measure of the dilation, may change with the accumulated plastic strain $\gamma$, whose evolution is related to the plastic strain rate by the equation

$$
\partial_{t} \gamma=\sqrt{2}\left|\operatorname{dev} \mathbf{V}^{(\mathrm{p})}\right| \text {. }
$$

We can eliminate $\mu$ from Eq. (6) by observing that (7) and (8) imply

$$
\mu=\partial_{t} \gamma / 2 \geq 0 \text {. }
$$

Plastic deformations occur only when the material is at yield, that is, the stress satisfies the plastic yield condition

$$
\tau=f(\sigma, \gamma)
$$


where $\sigma$ and $\tau$ are the mean and shear stresses:

$$
\sigma=\left(\sigma_{1}+\sigma_{2}\right) / 2, \quad \tau=\left(\sigma_{1}-\sigma_{2}\right) / 2 .
$$

We assume as typical behavior of the yield function that $f, f_{\sigma}$ and $f_{\gamma}$ are positive.

REMARK 1. A flow rule is said to be associated if the plastic deformation rate $\mathbf{V}^{(\mathrm{p})}$ is normal to the yield surface in stress space. In the case of (6), (10), associativity corresponds to having $\beta=f_{\sigma}$.

We differentiate (10) to obtain an expression for the rate of change of $\gamma$ in terms of the time derivatives of the mean and shear stresses:

$$
\partial_{t} \gamma= \begin{cases}{\left[\left(\partial_{t} \tau-f_{\sigma} \partial_{t} \sigma\right) / f_{\gamma}\right]_{+}} & \text {if } \tau=f(\sigma, \gamma) \\ 0 & \text { if } \tau<f(\sigma, \gamma)\end{cases}
$$

We combine (4), (5), (6), and (9) into a single constitutive relation, and with (1), (2), write the system of equations governing the deformation as

$$
\begin{aligned}
& \text { (a) } \partial_{t} \rho+\rho \nabla \cdot \overrightarrow{\mathbf{v}}=0, \\
& \text { (b) } \rho \partial_{t} \overrightarrow{\mathbf{v}}+\nabla \cdot \mathbf{T}=0, \\
& \text { (c) } \frac{1}{2} \Psi(\mathbf{T}) \partial_{t} \gamma+\frac{1}{2 G}\left(\partial_{t} \mathbf{T}-\nu\left(\operatorname{Tr} \partial_{t} \mathbf{T}\right) \mathbf{I}\right)+\mathbf{V}=0 .
\end{aligned}
$$

We wish to write the above system in a vector form more suitable for the study of its characteristic speeds. With that in mind, we write a symmetric $2 \times 2$ matrix $\mathbf{D}=\left(D_{i j}\right)$ in vector form as $\overrightarrow{\mathbf{D}}=\left(D_{11}, D_{22}, D_{12}\right)^{\mathrm{T}}$. We use this notation, along with (3), to rewrite (13) as a system of semilinear equations,

$$
\begin{aligned}
& \text { (a) } \partial_{t} \rho+\rho \nabla \cdot \overrightarrow{\mathbf{v}}=0 \\
& \text { (b) } \rho \partial_{t} \overrightarrow{\mathbf{v}}+\mathbf{A}_{1} \partial_{x} \overrightarrow{\mathbf{T}}+\mathbf{A}_{2} \partial_{y} \overrightarrow{\mathbf{T}}=0 \\
& \text { (c) } \vec{\Psi}(\overrightarrow{\mathbf{T}}) \partial_{t} \gamma / 2+\mathbf{C} \partial_{t} \overrightarrow{\mathbf{T}}+\widetilde{\mathbf{A}}_{1} \partial_{x} \overrightarrow{\mathbf{v}}+\widetilde{\mathbf{A}}_{2} \partial_{y} \overrightarrow{\mathbf{v}}=0
\end{aligned}
$$

where the coefficient matrices are

$$
\mathbf{A}_{1}=\left[\begin{array}{lll}
1 & 0 & 0 \\
0 & 0 & 1
\end{array}\right], \quad \mathbf{A}_{2}=\left[\begin{array}{lll}
0 & 0 & 1 \\
0 & 1 & 0
\end{array}\right], \quad \widetilde{\mathbf{A}}_{1}=\left[\begin{array}{ll}
1 & 0 \\
0 & 0 \\
0 & \frac{1}{2}
\end{array}\right], \quad \widetilde{\mathbf{A}}_{2}=\left[\begin{array}{ll}
0 & 0 \\
0 & 1 \\
\frac{1}{2} & 0
\end{array}\right]
$$

and

$$
\mathbf{C}=\frac{1}{2 G}\left[\begin{array}{ccc}
1-\nu & -\nu & 0 \\
-\nu & 1-\nu & 0 \\
0 & 0 & 1
\end{array}\right]
$$

3. Change of variables. The characteristic speeds of system (14), (12) are more easily calculated for diagonal stress tensors $\left(T_{12}=0\right)$. We introduce new stress variables, similar to Sokolovskii's [13] choice of independent stress variables, $\overrightarrow{\mathbf{T}}_{*}=\left(\sigma_{1}, \sigma_{2}, \phi\right)^{\mathrm{T}}$, where $\phi=\theta\left(\sigma_{1}-\sigma_{2}\right)$ and $\theta$ is the counterclockwise angle that the major principal stress axis makes with respect to the $x$-axis. In the following lemma, we show that these variables are locally equivalent to the stress tensor expressed in terms of a coordinate system whose axes are aligned with the principal stress axes. 
Lemma 1. Suppose that $\theta=\theta_{0}$ at some point $P_{0}\left(x_{0}, y_{0}, t_{0}\right)$ and $\sigma_{1}\left(P_{0}\right) \neq \sigma_{2}\left(P_{0}\right)$. Let $\mathbf{T}_{0}=\mathbf{R}\left(\theta_{0}\right)^{-1} \mathbf{T R}\left(\theta_{0}\right)$ where

$$
\mathbf{R}(\theta)=\left[\begin{array}{cc}
\cos \theta & -\sin \theta \\
\sin \theta & \cos \theta
\end{array}\right]
$$

Then $\frac{d \overrightarrow{\mathbf{T}}_{0}}{d \overrightarrow{\mathbf{T}}_{*}}\left(P_{0}\right)=\mathbf{I}$.

Proof. Since $\mathbf{T}$ is expressed in terms of the principal stress as $\mathbf{T}=\mathbf{R}(\theta)\left[\begin{array}{cc}\sigma_{1} & 0 \\ 0 & \sigma_{2}\end{array}\right] \mathbf{R}(\theta)^{-1}$, we can write $\mathbf{T}_{0}=\mathbf{R}\left(\theta-\theta_{0}\right)\left[\begin{array}{cc}\sigma_{1} & 0 \\ 0 & \sigma_{2}\end{array}\right] \mathbf{R}\left(\theta-\theta_{0}\right)^{-1}$. We let $\omega=\theta-\theta_{0}$ and write

$$
\frac{d \mathbf{T}_{0}}{d \overrightarrow{\mathbf{T}}_{*}}\left(P_{0}\right)=\left.\frac{d}{d \overrightarrow{\mathbf{T}}_{*}}\left(\mathbf{R}(\omega)\left[\begin{array}{cc}
\sigma_{1} & 0 \\
0 & \sigma_{2}
\end{array}\right] \mathbf{R}(\omega)^{-1}\right)\right|_{\omega=0}
$$

We evaluate the derivatives with respect to each component of $\overrightarrow{\mathbf{T}}_{*}$ :

$$
\frac{\partial \mathbf{T}_{0}}{\partial \sigma_{1}}\left(P_{0}\right)=\left[\begin{array}{ll}
1 & 0 \\
0 & 0
\end{array}\right], \quad \frac{\partial \mathbf{T}_{0}}{\partial \sigma_{2}}\left(P_{0}\right)=\left[\begin{array}{ll}
0 & 0 \\
0 & 1
\end{array}\right],
$$

and

$$
\begin{aligned}
\frac{d \mathbf{T}_{0}}{d \phi}\left(P_{0}\right) & =\left.\frac{1}{\sigma_{1}-\sigma_{2}}\left(\mathbf{R}^{\prime}(\omega)\left[\begin{array}{cc}
\sigma_{1} & 0 \\
0 & \sigma_{2}
\end{array}\right] \mathbf{R}(\omega)^{-1}+\mathbf{R}(\omega)\left[\begin{array}{cc}
\sigma_{1} & 0 \\
0 & \sigma_{2}
\end{array}\right]\left(\mathbf{R}(\omega)^{-1}\right)^{\prime}\right)\right|_{\omega=0} \\
& =\left[\begin{array}{ll}
0 & 1 \\
1 & 0
\end{array}\right] \text { since } \sigma_{1}\left(P_{0}\right) \neq \sigma_{2}\left(P_{0}\right) .
\end{aligned}
$$

The lemma is proved by writing these derivatives in vector form.

For plastic deformations we always have $\tau>0$ (by (10)) and therefore $\sigma_{1} \neq \sigma_{2}$. In this case the hypotheses of Lemma 1 are satisfied and the change of variables is therefore well defined.

THEOREM 2. Wherever $\sigma_{1} \neq \sigma_{2},(14)$ is equivalent to

(a) $\partial_{t} \rho+\rho \nabla \cdot \overrightarrow{\mathbf{v}}=0$,

(b) $\rho \partial_{t} \overrightarrow{\mathbf{v}}+\mathbf{R}(\theta) \mathbf{L}(\theta) \partial_{x} \overrightarrow{\mathbf{T}}_{*}+\mathbf{R}(\theta) \mathbf{M}(\theta) \partial_{y} \overrightarrow{\mathbf{T}}_{*}=0$

(c) $\frac{1}{2} \vec{\Psi}\left(\left(\sigma_{1}, \sigma_{2}, 0\right)^{\mathrm{T}}\right) \partial_{t} \gamma+\mathbf{C} \partial_{t} \overrightarrow{\mathbf{T}}_{*}+\widetilde{\mathbf{L}}(\theta) \mathbf{R}(\theta)^{-1} \partial_{x} \overrightarrow{\mathbf{v}}+\widetilde{\mathbf{M}}(\theta) \mathbf{R}(\theta)^{-1} \partial_{y} \overrightarrow{\mathbf{v}}=0$,

where $\mathbf{L}(\theta)=\mathbf{A}_{1} \cos \theta-\mathbf{A}_{2} \sin \theta, \mathbf{M}(\theta)=\mathbf{A}_{1} \sin \theta+\mathbf{A}_{2} \cos \theta, \widetilde{\mathbf{L}}(\theta)=\widetilde{\mathbf{A}}_{1} \cos \theta-\widetilde{\mathbf{A}}_{2} \sin \theta$, and $\widetilde{\mathbf{M}}(\theta)=\widetilde{\mathbf{A}}_{1} \sin \theta+\widetilde{\mathbf{A}}_{2} \cos \theta$.

Proof. Let $\theta_{0}, P_{0}$ and $\mathbf{T}_{0}$ be as in Lemma 1 . We define an auxiliary frame of reference, $(\bar{x}, \bar{y})^{\mathrm{T}}=\mathbf{R}\left(\theta_{0}\right)^{-1}(x, y)^{\mathrm{T}}$, and the velocity in these coordinates, $\overrightarrow{\mathbf{v}}_{0}=\mathbf{R}\left(\theta_{0}\right)^{-1} \overrightarrow{\mathbf{v}}$. Then $(14 \mathrm{~b}, \mathrm{c})$ implies

$$
\begin{gathered}
\rho \partial_{t} \overrightarrow{\mathbf{v}}_{0}+\mathbf{A}_{1} \partial_{\bar{x}} \overrightarrow{\mathbf{T}}_{0}+\mathbf{A}_{2} \partial_{\bar{y}} \overrightarrow{\mathbf{T}}_{0}=0 \\
\vec{\Psi}\left(\overrightarrow{\mathbf{T}}_{0}\right) \partial_{t} \gamma / 2+\mathbf{C} \partial_{t} \overrightarrow{\mathbf{T}}_{0}+\widetilde{\mathbf{A}}_{1} \partial_{\bar{x}} \overrightarrow{\mathbf{v}}_{0}+\widetilde{\mathbf{A}}_{2} \partial_{\bar{y}} \overrightarrow{\mathbf{v}}_{0}=0
\end{gathered}
$$


We invert the change of coordinates and velocity variables,

$$
\begin{aligned}
\rho \mathbf{R}\left(\theta_{0}\right)^{-1} \partial_{t} \overrightarrow{\mathbf{v}}+\left(\mathbf{A}_{1} \cos \theta_{0}-\mathbf{A}_{2} \sin \theta_{0}\right) \partial_{x} \overrightarrow{\mathbf{T}}_{0}+\left(\mathbf{A}_{1} \sin \theta_{0}+\mathbf{A}_{2} \cos \theta_{0}\right) \partial_{y} \overrightarrow{\mathbf{T}}_{0}=0, \\
\vec{\Psi}\left(\left(\sigma_{1}, \sigma_{2}, 0\right)^{\mathrm{T}}\right) \partial_{t} \gamma / 2+\mathbf{C} \partial_{t} \overrightarrow{\mathbf{T}}_{0}+\left(\widetilde{\mathbf{A}}_{1} \cos \theta_{0}-\widetilde{\mathbf{A}}_{2} \sin \theta_{0}\right) \mathbf{R}\left(\theta_{0}\right)^{-1} \partial_{x} \overrightarrow{\mathbf{v}} \\
+\left(\widetilde{\mathbf{A}}_{1} \sin \theta_{0}+\widetilde{\mathbf{A}}_{2} \cos \theta_{0}\right) \mathbf{R}\left(\theta_{0}\right)^{-1} \partial_{y} \overrightarrow{\mathbf{v}}=0,
\end{aligned}
$$

and apply Lemma 1 at $P_{0}$,

$$
\begin{gathered}
\rho \partial_{t} \overrightarrow{\mathbf{v}}+\mathbf{R}\left(\theta_{0}\right) \mathbf{L}\left(\theta_{0}\right) \partial_{x} \overrightarrow{\mathbf{T}}_{*}+\mathbf{R}\left(\theta_{0}\right) \mathbf{M}\left(\theta_{0}\right) \partial_{y} \overrightarrow{\mathbf{T}}_{*}=0, \\
\vec{\Psi}\left(\left(\sigma_{1}, \sigma_{2}, 0\right)^{\mathrm{T}}\right) \partial_{t} \gamma / 2+\mathbf{C} \partial_{t} \overrightarrow{\mathbf{T}}_{*}+\widetilde{\mathbf{L}}\left(\theta_{0}\right) \mathbf{R}\left(\theta_{0}\right)^{-1} \partial_{x} \overrightarrow{\mathbf{v}}+\widetilde{\mathbf{M}}\left(\theta_{0}\right) \mathbf{R}\left(\theta_{0}\right)^{-1} \partial_{y} \overrightarrow{\mathbf{v}}=0 .
\end{gathered}
$$

This shows that $(15 \mathrm{~b}, \mathrm{c})$ holds wherever $\sigma_{1} \neq \sigma_{2}$.

4. Characteristic speeds. In this section, we use the previous change of variables to calculate the characteristic speeds of plane wave solutions to system (14), (12) or, equivalently, to (15), (12). We consider solutions that depend only on $x$ and $t$ (rotational invariance of the equations assures the same result for other directions in the $(x, y)$-plane). So we write the one-dimensional system

$$
\begin{aligned}
& \text { (a) } \partial_{t} \rho+\rho \partial_{x} v_{1}=0 \\
& \text { (b) } \rho \partial_{t} \overrightarrow{\mathbf{v}}+\mathbf{A}_{1} \partial_{x} \overrightarrow{\mathbf{T}}=0 \\
& \text { (c) } \frac{1}{2} \vec{\Psi}(\overrightarrow{\mathbf{T}}) \partial_{t} \gamma+\mathbf{C} \partial_{t} \overrightarrow{\mathbf{T}}+\widetilde{\mathbf{A}}_{1} \partial_{x} \overrightarrow{\mathbf{v}}=0
\end{aligned}
$$

where $\partial_{t} \gamma$ is given by (12). In the case of elastic deformation, (16) reduces to the linear system

$$
\begin{aligned}
& \text { (a) } \partial_{t} \rho+\rho \partial_{x} v_{1}=0 \\
& \text { (b) } \rho \partial_{t} \overrightarrow{\mathbf{v}}+\mathbf{A}_{1} \partial_{x} \overrightarrow{\mathbf{T}}=0, \\
& \text { (c) } \partial_{t} \overrightarrow{\mathbf{T}}+\mathbf{C}^{-1} \widetilde{\mathbf{A}}_{1} \partial_{x} \overrightarrow{\mathbf{v}}=0 .
\end{aligned}
$$

As shown in [11], the characteristic speeds of the system (17) are $0, \pm \sqrt{\eta_{1}}, \pm \sqrt{\eta_{2}}$, where $\eta_{1}$ and $\eta_{2}$ are the eigenvalues of $\rho^{-1} \mathbf{A}_{1} \mathbf{C}^{-1} \widetilde{\mathbf{A}}_{1}$ :

$$
\eta_{1}=\frac{2 G}{\rho}\left(\frac{1-\nu}{1-2 \nu}\right), \quad \eta_{2}=\frac{G}{\rho}
$$

(we make the standard assumption that $1-2 \nu>0$ ).

As we noted before, in the case of plastic deformation, $\tau>0$ and $\sigma_{1} \neq \sigma_{2}$, so that we may invoke Theorem 2. This allows us to write system (16) for plastic deformations as

(a) $\partial_{t} \rho+\rho \partial_{x} v_{1}=0$

(b) $\rho \partial_{t} \overrightarrow{\mathbf{v}}+\mathbf{R}(\theta) \mathbf{L}(\theta) \partial_{x} \overrightarrow{\mathbf{T}}_{*}=0$

(c) $\quad\left[\frac{1}{2} \vec{\Psi}\left(\left(\sigma_{1}, \sigma_{2}, 0\right)^{\mathrm{T}}\right)\left(\frac{d \gamma}{d \overrightarrow{\mathbf{T}}_{*}}\right)^{\mathrm{T}}+\mathbf{C}\right] \partial_{t} \overrightarrow{\mathbf{T}}_{*}+\widetilde{\mathbf{L}}(\theta) \mathbf{R}(\theta)^{-1} \partial_{x} \overrightarrow{\mathbf{v}}=0$. 
THEOREM 3. The characteristic speeds of $(19)$ are $0, \pm \sqrt{\mu_{1}}, \pm \sqrt{\mu_{2}}$, where

$$
\begin{aligned}
& \mu_{1}=\frac{G}{\rho}\left(1+\frac{H}{2 K}+\sqrt{\frac{H^{2}}{4 K^{2}}+\frac{\sin ^{2} 2 \theta}{K}}\right) \\
& \mu_{2}=\frac{G}{\rho}\left(1+\frac{H}{2 K}-\sqrt{\frac{H^{2}}{4 K^{2}}+\frac{\sin ^{2} 2 \theta}{K}}\right),
\end{aligned}
$$

$H=\left(\beta+f_{\sigma}\right) \cos 2 \theta+c+2 \nu, K=(1-2 \nu)(c+1)+\beta f_{\sigma}$, and $c=f_{\gamma} / G$.

Proof. As shown in [11], $\mu_{1}$ and $\mu_{2}$ are the eigenvalues of $\mathbf{\Gamma}=\rho^{-1} \mathbf{L B}^{-1} \widetilde{\mathbf{L}}$, where

$$
\mathbf{B}=\frac{1}{2} \vec{\Psi}\left(\left(\sigma_{1}, \sigma_{2}, 0\right)^{\mathrm{T}}\right)\left(\frac{d \gamma}{d \overrightarrow{\mathbf{T}}_{*}}\right)^{\mathrm{T}}+\mathbf{C} .
$$

We notice that for a diagonal stress, (7) is rewritten in vector form as

$$
\vec{\Psi}\left(\left(\sigma_{1}, \sigma_{2}, 0\right)^{\mathrm{T}}\right)=\left(\begin{array}{c}
1-\beta \\
-(1+\beta) \\
0
\end{array}\right) .
$$

Also, differentiating (10) with respect to $\overrightarrow{\mathbf{T}}_{*}$, we obtain

$$
\frac{d \gamma}{d \overrightarrow{\mathbf{T}}_{*}}=\frac{1}{2 f_{\gamma}}\left(\begin{array}{c}
1-f_{\sigma} \\
-\left(1+f_{\sigma}\right) \\
0
\end{array}\right) .
$$

Using (20) and (21), we notice that $\mathbf{B}$ has a block structure that simplifies the calculation of its inverse:

$$
\mathbf{B}=\frac{1}{4 f_{\gamma}}\left[\begin{array}{ccc}
b_{11} & -b_{12} & 0 \\
-b_{21} & b_{22} & 0 \\
0 & 0 & 2 c
\end{array}\right],
$$

where $b_{11}=(1-\beta)\left(1-f_{\sigma}\right)+2 c(1-\nu), b_{22}=(1+\beta)\left(1+f_{\sigma}\right)+2 c(1-\nu), b_{12}=$ $(1-\beta)\left(1+f_{\sigma}\right)+2 c \nu$, and $b_{21}=(1+\beta)\left(1-f_{\sigma}\right)+2 c \nu$. We write the inverse of $\mathbf{B}$ as

$$
\mathbf{B}^{-1}=\frac{G}{K}\left[\begin{array}{ccc}
b_{22} & b_{12} & 0 \\
b_{21} & b_{11} & 0 \\
0 & 0 & 2 K
\end{array}\right] .
$$

(We notice that $K$ is positive by (15) of [11].) Finally, we can write the matrix $\Gamma$ as

$$
\Gamma=\frac{G}{\rho K}\left[\begin{array}{cc}
b_{22} \cos ^{2} \theta+K \sin ^{2} \theta & -\left(b_{12}+K\right) \cos \theta \sin \theta \\
-\left(b_{21}+K\right) \cos \theta \sin \theta & b_{11} \sin ^{2} \theta+K \cos ^{2} \theta
\end{array}\right],
$$

which allows for an easy calculation of the eigenvalues $\mu_{1}$ and $\mu_{2}$.

For convenience in the following calculations, we write $\xi_{1}=\cos \theta$ and $\xi_{2}=\sin \theta$. We will express $\mu_{i}$ in terms of the trace and determinant of $\boldsymbol{\Gamma}$,

$$
\mu=\operatorname{Tr} \boldsymbol{\Gamma} / 2 \pm \sqrt{(\operatorname{Tr} \boldsymbol{\Gamma})^{2} / 4-\operatorname{det} \boldsymbol{\Gamma}} .
$$


We notice that $K=\left(b_{22}+b_{11}\right) / 2-(c+2 \nu)$ and $H=\left(b_{22}-b_{11}\right)\left(\xi_{1}^{2}-\xi_{2}^{2}\right) / 2+c+2 \nu$, which combines to

$$
K+H=b_{22} \xi_{1}^{2}+b_{11} \xi_{2}^{2}
$$

Now we write

$$
\operatorname{Tr} \boldsymbol{\Gamma}=\frac{G}{\rho K}\left(b_{22} \xi_{1}^{2}+b_{11} \xi_{2}^{2}+K\right)=\frac{G}{\rho K}(2 K+H)
$$

and

$$
\begin{aligned}
(\operatorname{Tr} \boldsymbol{\Gamma})^{2} / 4-\operatorname{det} \boldsymbol{\Gamma}= & \frac{G^{2}}{\rho^{2} K^{2}}\left[K^{2}+K H+H^{2} / 4\right] \\
& -\frac{G^{2}}{\rho^{2} K^{2}}\left[\left(b_{22} \xi_{1}^{2}+K \xi_{2}^{2}\right)\left(b_{11} \xi_{2}^{2}+K \xi_{1}^{2}\right)-\left(b_{12}+K\right)\left(b_{21}+K\right) \xi_{1}^{2} \xi_{2}^{2}\right] \\
= & \frac{G^{2}}{\rho^{2} K^{2}}\left[H^{2} / 4+K(K+H)\right. \\
& \left.-K\left(b_{22} \xi_{1}^{4}+b_{11} \xi_{2}^{4}\right)+K\left(b_{12}+b_{21}\right) \xi_{1}^{2} \xi_{2}^{2}-\left(b_{11} b_{22}-b_{12} b_{21}\right) \xi_{1}^{2} \xi_{2}^{2}\right]
\end{aligned}
$$

Applying (22) to the second term, noticing that $b_{11} b_{22}-b_{12} b_{21}=4 c K$, and using the definition of the $b_{i j}$ 's, we have

$$
\begin{aligned}
\frac{1}{4}(\operatorname{Tr} \boldsymbol{\Gamma})^{2}-\operatorname{det} \boldsymbol{\Gamma} & =\frac{G^{2}}{\rho^{2} K^{2}}\left[H^{2} / 4+K\left(b_{22}+b_{11}+b_{12}+b_{21}\right) \xi_{1}^{2} \xi_{2}^{2}-4 c K \xi_{1}^{2} \xi_{2}^{2}\right] \\
& =\frac{G^{2}}{\rho^{2} K^{2}}\left(H^{2} / 4+4 K \xi_{1}^{2} \xi_{2}^{2}\right) .
\end{aligned}
$$

The theorem now follows from (23) and (24).

REMARK 2. A discussion of the order of the characteristic speeds of system (19) only makes sense if the system is hyperbolic, i.e., if $\mu_{1}, \mu_{2} \geq 0$. It is shown in [11] that hyperbolicity is equivalent to $c \geq\left(\beta-f_{\sigma}\right)^{2} /(8(1-\nu))$. Loss of hyperbolicity has the implication that the linearized equations are ill-posed in the sense of Hadamard, and is widely recognized as an indication of the formation of shear bands (cf. [7], [9], [11]).

THEOREM 4. For slow waves, elastic waves always travel faster than plastic waves. Moreover, we have $\mu_{2} \leq \eta_{2} \leq \mu_{1}$.

Proof. It is clear from Theorem 3 that $\mu_{2} \leq \frac{G}{\rho} \leq \mu_{1}$, and since $\eta_{2}=G / \rho$ (from (18)), the theorem follows.

Next we show that in general we cannot expect that fast elastic waves travel faster than fast plastic waves. In particular, we show that there is a well-defined region in the stress space for which the fast elastic waves are slower than the fast plastic waves (see Figure 1).

TheOREm 5. For values of the stress tensor in the region defined by

$$
\frac{\min \left\{\beta, f_{\sigma}\right\}}{1-2 \nu}<\cos 2 \theta<\frac{\max \left\{\beta, f_{\sigma}\right\}}{1-2 \nu},
$$

the characteristic speeds of waves of the fast family satisfy $\mu_{1}>\eta_{1}$. Further, if we assume $\beta>0$ (dilation) then $\mu_{1} \leq \eta_{1}$ in regions where (25) fails. 


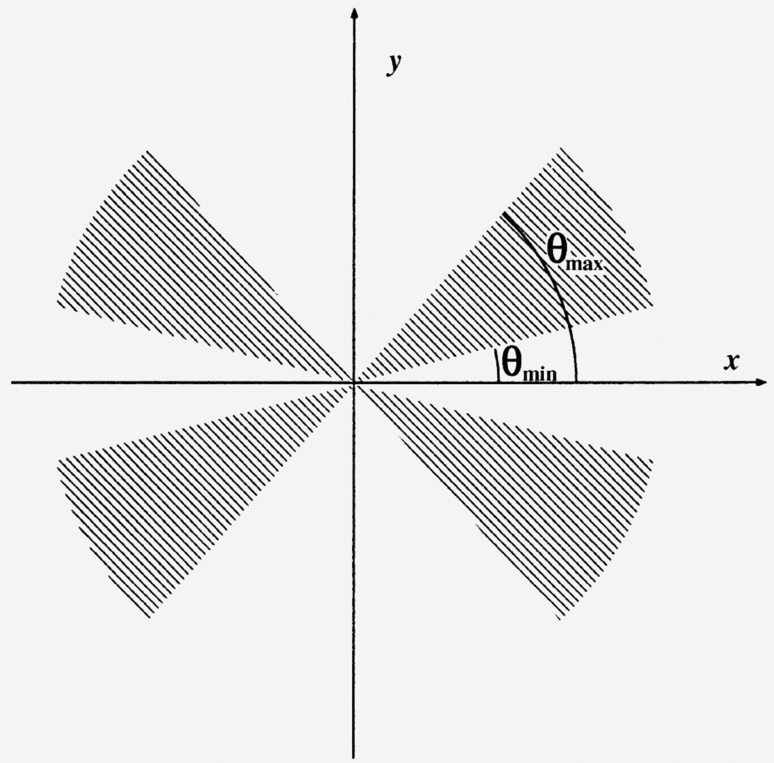

FIG. 1. Values of $\theta$ for which $\mu_{1}>\eta_{1}$

Proof. By (18) and Theorem $3, \mu_{1} \leq \eta_{1}$ if and only if

$$
\left(H^{2}+4 K \sin ^{2} 2 \theta\right)^{1 / 2} \leq \frac{2 K}{1-2 \nu}-H .
$$

This implies $H^{2}+4 K \sin ^{2}(2 \theta) \leq\left(\frac{2 K}{1-2 \nu}-H\right)^{2}$, which is equivalent to

$$
\begin{aligned}
\sin ^{2} 2 \theta \leq & \frac{K}{(1-2 \nu)^{2}}-\frac{H}{1-2 \nu}=1-\frac{\left(\beta+f_{\sigma}\right) \cos 2 \theta}{1-2 \nu}+\frac{\beta f_{\sigma}}{(1-2 \nu)^{2}} \\
& \Leftrightarrow \cos ^{2} 2 \theta-\frac{\left(\beta+f_{\sigma}\right) \cos 2 \theta}{1-2 \nu}+\frac{\beta f_{\sigma}}{(1-2 \nu)^{2}} \geq 0 \\
& \Leftrightarrow\left(\cos 2 \theta-\frac{\beta}{1-2 \nu}\right)\left(\cos 2 \theta-\frac{f_{\sigma}}{1-2 \nu}\right) \geq 0,
\end{aligned}
$$

which proves the first part of the theorem. Clearly the converse will hold if the right side of (26) is positive. If $\beta>0$ then

$$
\begin{aligned}
& c+1-2 \nu+(1-\beta)\left(1-f_{\sigma}\right)+\beta f_{\sigma}\left(\frac{1+2 \nu}{1-2 \nu}\right)>0 \\
& \Rightarrow 2(c+1)+\frac{2 \beta f_{\sigma}}{1-2 \nu}>\beta+f_{\sigma}+c+2 \nu \geq H
\end{aligned}
$$

and so $\frac{2 K}{1-2 \nu}-H>0$.

Notice that the region described by (25) vanishes only for associated flows, i.e., $\beta=f_{\sigma}$.

A different difficulty arises when the fast and slow plastic waves travel at the same speed, a phenomenon that is related to the occurrence of the flutter instability (cf. [1], [2], [9]). From the previous theorems, this is only possible if $\mu_{2}=\mu_{1}=G / \rho$. In the following theorem, we express this identity in terms of the parameters. 
THEOREM 6. The values of stress for which the speeds of the fast and slow plastic waves coincide are exactly those that satisfy $\beta+f_{\sigma}=(-1)^{n+1}(c+2 \nu)$ and $\theta=n \pi / 2, n \in \mathbf{Z}$.

Proof. From Theorem 3, $\mu_{1}=\mu_{2} \Leftrightarrow\left(H^{2}+4 K \sin ^{2} 2 \theta\right) /\left(4 K^{2}\right)=0 \Leftrightarrow H=0$ and $\theta=n \pi / 2, n \in \mathbf{Z} \Leftrightarrow\left(\beta+f_{\sigma}\right)(-1)^{n}+(c+2 \nu)=0$, from which the theorem follows.

This result was already established in a weaker form in [2] and in a similarly general form in [1]. We include it in this paper for the sake of completeness and because of the simplicity of our proof.

5. Inclusion of the rotational terms. In system (13), the rotational terms of the Jaumann stress rate were ignored, that is, $\partial_{t} \mathbf{T}-\mathbf{T} \boldsymbol{\Omega}-(\mathbf{T} \boldsymbol{\Omega})^{\mathrm{T}}$ was replaced by the time derivative of stress, $\partial_{t} \mathbf{T}$. In this section, we show that the results of Section 4 remain qualitatively the same when the rotational terms are retained.

As before, we continue to ignore the convective terms $\overrightarrow{\mathbf{v}} \cdot \nabla$ in the material derivative, since these will merely shift all the characteristic speeds by $v_{1}$.

Replacing $\partial_{t} \mathbf{T}$ by the Jaumann stress rate in the constitutive relation (13c) gives

$$
\frac{1}{2} \Psi(\mathbf{T}) \partial_{t} \gamma+\frac{1}{2 G}\left(\partial_{t} \mathbf{T}-\nu\left(\operatorname{Tr} \partial_{t} \mathbf{T}\right) \mathbf{I}-\mathbf{T} \boldsymbol{\Omega}-(\mathbf{T} \boldsymbol{\Omega})^{\mathrm{T}}\right)+\mathbf{V}=0
$$

where $\boldsymbol{\Omega}=\frac{1}{2}\left(\nabla \overrightarrow{\mathbf{v}}^{\mathrm{T}}-\left(\nabla \overrightarrow{\mathbf{v}}^{\mathrm{T}}\right)^{\mathrm{T}}\right)$. As in $(14 \mathrm{c})$, we express this relation in vector form:

$$
\frac{1}{2} \vec{\Psi}(\overrightarrow{\mathbf{T}}) \partial_{t} \gamma+\mathbf{C} \partial_{t} \overrightarrow{\mathbf{T}}+\frac{1}{2 G}\left(\begin{array}{c}
-T_{12} \\
T_{12} \\
\frac{1}{2}\left(T_{11}-T_{22}\right)
\end{array}\right)\left(\partial_{y} v_{1}-\partial_{x} v_{2}\right)+\widetilde{\mathbf{A}} \partial_{x} \overrightarrow{\mathbf{v}}+\widetilde{\mathbf{A}} \partial_{y} \overrightarrow{\mathbf{v}}=0
$$

We now write this in terms of the principal stresses as in (15c):

$$
\begin{aligned}
& \frac{1}{2} \vec{\Psi}\left(\left(\sigma_{1}, \sigma_{2}, 0\right)^{\mathrm{T}}\right) \partial_{t} \gamma+\mathbf{C} \partial_{t} \overrightarrow{\mathbf{T}}_{*}+\frac{1}{2 G}\left(\begin{array}{l}
0 \\
0 \\
\tau
\end{array}\right)\left(\partial_{y} v_{1}-\partial_{x} v_{2}\right)+\widetilde{\mathbf{L}}(\theta) \mathbf{R}(\theta)^{-1} \partial_{x} \overrightarrow{\mathbf{v}} \\
&+\widetilde{\mathbf{M}}(\theta) \mathbf{R}(\theta)^{-1} \partial_{y} \overrightarrow{\mathbf{v}}=0 .
\end{aligned}
$$

For planar waves in the $x$-direction, the constitutive relation for elastic deformations takes the form

$$
\mathbf{C} \partial_{t} \overrightarrow{\mathbf{T}}_{*}-\frac{1}{2 G}\left(\begin{array}{c}
0 \\
0 \\
\tau
\end{array}\right) \partial_{x} v_{2}+\widetilde{\mathbf{L}}(\theta) \mathbf{R}(\theta)^{-1} \partial_{x} \overrightarrow{\mathbf{v}}=0
$$

and the constitutive relation for plastic deformations takes the form

$$
\mathbf{B} \partial_{t} \overrightarrow{\mathbf{T}}_{*}-\frac{1}{2 G}\left(\begin{array}{l}
0 \\
0 \\
\tau
\end{array}\right) \partial_{x} v_{2}+\widetilde{\mathbf{L}}(\theta) \mathbf{R}(\theta)^{-1} \partial_{x} \overrightarrow{\mathbf{v}}=0
$$


In Appendix A, we give typical values of the parameters, for which $\tau / G=O\left(10^{-2}\right)$. In part of the analysis that follows, we assume that $\tau<G$.

THEOREM 7. The characteristic speeds of the modified system $(19 \mathrm{a}, \mathrm{b}),(28)$ are $0, \pm \sqrt{\tilde{\mu}_{1}}$, $\pm \sqrt{\tilde{\mu}_{2}}$, where

$$
\begin{aligned}
& \tilde{\mu}_{1}=\frac{G}{\rho}\left[1+\frac{H}{2 K}+\frac{\tau}{2 G} \cos 2 \theta+\left(\frac{1}{4}\left(\frac{H}{K}+\frac{\tau}{G} \cos 2 \theta\right)^{2}+\frac{1}{K}\left(1+\frac{\tau f_{\sigma}}{G}\right) \sin ^{2} 2 \theta\right)^{1 / 2}\right], \\
& \tilde{\mu}_{2}=\frac{G}{\rho}\left[1+\frac{H}{2 K}+\frac{\tau}{2 G} \cos 2 \theta-\left(\frac{1}{4}\left(\frac{H}{K}+\frac{\tau}{G} \cos 2 \theta\right)^{2}+\frac{1}{K}\left(1+\frac{\tau f_{\sigma}}{G}\right) \sin ^{2} 2 \theta\right)^{1 / 2}\right],
\end{aligned}
$$

$H=\left(\beta+f_{\sigma}\right) \cos 2 \theta+c+2 \nu$ and $K=(1-2 \nu)(c+1)+\beta f_{\sigma}$.

Proof. As in Theorem 3, $\tilde{\mu}_{1}$ and $\tilde{\mu}_{2}$ are the eigenvalues of

$$
\widetilde{\boldsymbol{\Gamma}}=\frac{G}{\rho K}\left[\begin{array}{cc}
b_{22} \cos ^{2} \theta+K\left(1+\frac{\tau}{G}\right) \sin ^{2} \theta & -\left(b_{12}+K\left(1-\frac{\tau}{G}\right)\right) \cos \theta \sin \theta \\
-\left(b_{21}+K\left(1+\frac{\tau}{G}\right)\right) \cos \theta \sin \theta & b_{11} \sin ^{2} \theta+K\left(1-\frac{\tau}{G}\right) \cos ^{2} \theta
\end{array}\right] .
$$

We write $\widetilde{\boldsymbol{\Gamma}}$ in terms of $\boldsymbol{\Gamma}$, of the previous section, as

$$
\widetilde{\Gamma}=\Gamma+\frac{\tau}{\rho}\left[\begin{array}{cc}
\xi_{2}^{2} & \xi_{1} \xi_{2} \\
-\xi_{1} \xi_{2} & \xi_{1}^{2}
\end{array}\right]
$$

where $\xi_{1}=\cos \theta$ and $\xi_{2}=\sin \theta$, and express the eigenvalues of $\widetilde{\Gamma}$ in terms of its trace and determinant. We have that

$$
\operatorname{Tr} \widetilde{\boldsymbol{\Gamma}}=\operatorname{Tr} \boldsymbol{\Gamma}-\frac{\tau}{\rho}\left(\xi_{1}^{2}-\xi_{2}^{2}\right)=\operatorname{Tr} \boldsymbol{\Gamma}-\frac{\tau}{\rho} \cos 2 \theta
$$

and

$$
\begin{aligned}
\operatorname{det} \widetilde{\boldsymbol{\Gamma}} & =\operatorname{det} \boldsymbol{\Gamma}-\frac{\tau}{\rho}\left(\xi_{1}^{2} \Gamma_{11}-\xi_{2}^{2} \Gamma_{22}+\xi_{1} \xi_{2}\left(\Gamma_{21}-\Gamma_{12}\right)\right) \\
& =\operatorname{det} \boldsymbol{\Gamma}-\frac{\tau G}{\rho^{2} K}\left(\beta+f_{\sigma}+\left(1+\beta f_{\sigma}+2 c(1-\nu)\right)\left(\xi_{1}^{2}-\xi_{2}^{2}\right)-4 \beta \xi_{1}^{2} \xi_{2}^{2}\right) \\
& =\operatorname{det} \boldsymbol{\Gamma}-\frac{\tau G}{\rho^{2} K}\left(\beta+f_{\sigma}+(K+c+2 \nu) \cos 2 \theta-\beta \sin ^{2} 2 \theta\right) \\
& =\operatorname{det} \boldsymbol{\Gamma}-\frac{\tau G}{\rho^{2} K}\left((K+H) \cos 2 \theta+f_{\sigma} \sin ^{2} 2 \theta\right)
\end{aligned}
$$


As before, we combine these,

$$
\begin{aligned}
\frac{1}{4}(\operatorname{Tr} \widetilde{\boldsymbol{\Gamma}})^{2}-\operatorname{det} \widetilde{\boldsymbol{\Gamma}}= & \frac{1}{4}\left(\operatorname{Tr} \boldsymbol{\Gamma}-\frac{\tau}{\rho} \cos 2 \theta\right)^{2}-\operatorname{det} \boldsymbol{\Gamma}+\frac{\tau G}{\rho^{2}}\left(1+\frac{H}{K}-\frac{1}{K} \sin ^{2} 2 \theta\right) \\
= & \frac{G^{2}}{\rho^{2}}\left[\frac{1}{4}\left(2+\frac{H}{K}-\frac{\tau}{G} \cos 2 \theta\right)^{2}-\operatorname{det} \boldsymbol{\Gamma}\right. \\
& \left.+\frac{\tau}{G}\left(\left(1+\frac{H}{K}\right) \cos 2 \theta+\frac{f_{\sigma}}{K} \sin ^{2} 2 \theta\right)\right] \\
= & \frac{G^{2}}{\rho^{2}}\left[\frac{1}{4}\left(\frac{H}{K}-\frac{\tau}{G} \cos 2 \theta\right)^{2}+\frac{1}{K} \sin ^{2} 2 \theta-\frac{\tau}{G} \cos 2 \theta\right. \\
& \left.+\frac{\tau}{G}\left(\left(1+\frac{H}{K}\right) \cos 2 \theta+\frac{f_{\sigma}}{K} \sin ^{2} 2 \theta\right)\right] \\
= & \frac{G^{2}}{\rho^{2}}\left[\frac{1}{4}\left(\frac{H}{K}-\frac{\tau}{G} \cos 2 \theta\right)^{2}+\frac{1}{K}\left(1+\frac{\tau f_{\sigma}}{G}\right) \sin ^{2} 2 \theta+\frac{\tau H}{G K} \cos 2 \theta\right] \\
= & \frac{G^{2}}{\rho^{2}}\left[\frac{1}{4}\left(\frac{H}{K}+\frac{\tau}{G} \cos 2 \theta\right)^{2}+\frac{1}{K}\left(1+\frac{\tau f_{\sigma}}{G}\right) \sin ^{2} 2 \theta\right] .
\end{aligned}
$$

The theorem follows from this and the equation for $\operatorname{Tr} \widetilde{\Gamma}$.

TheOREM 8. The characteristic speeds of the modified system $(19 \mathrm{a}, \mathrm{b}),(27)$ are $0, \pm \sqrt{\tilde{\eta}_{1}}$, $\pm \sqrt{\tilde{\eta}_{2}}$, where

$$
\begin{gathered}
\tilde{\eta}_{1}=\frac{G}{\rho}\left[\frac{2(1-\nu)}{1-2 \nu}+\frac{\tau}{G} \cos 2 \theta\right], \\
\tilde{\eta}_{2}=\frac{G}{\rho} .
\end{gathered}
$$

Proof. Notice from (12) that $\lim _{f_{\gamma} \rightarrow \infty} \mathbf{B}=\mathbf{C}$ which implies that $\tilde{\eta}_{i}=\lim _{f_{\gamma} \rightarrow \infty} \tilde{\mu}_{i}$, $i=1,2 ;$ so the result follows from Theorem 7 .

Theorem 9. Suppose $\tau<G$.

(a) For all values of stress, $\tilde{\mu}_{2} \leq \tilde{\eta}_{2} \leq \tilde{\mu}_{1}$.

(b) For $\tilde{\beta}=\beta+\frac{\tau\left(\beta^{2}-(1-2 \nu)^{2}\right)}{G-\tau \beta}$, if the stress tensor is in the region defined by

$$
\frac{\min \left\{\tilde{\beta}, f_{\sigma}\right\}}{1-2 \nu}<\cos 2 \theta<\frac{\max \left\{\tilde{\beta}, f_{\sigma}\right\}}{1-2 \nu}
$$

then $\tilde{\mu}_{1}>\tilde{\eta}_{1}$.

Proof. Part (a) follows immediately from Theorems 7 and 8. We notice that $\tilde{\mu}_{1} \leq \tilde{\eta}_{1}$ only if

$$
\begin{gathered}
\left(H+\frac{\tau K}{G} \cos 2 \theta\right)^{2}+4 K\left(1+\frac{\tau f_{\sigma}}{G}\right) \sin ^{2} 2 \theta \leq\left(\frac{2 K}{1-2 \nu}-H+\frac{\tau K}{G} \cos 2 \theta\right)^{2} \\
\Leftrightarrow\left(\cos 2 \theta-\frac{f_{\sigma}}{1-2 \nu}\right)\left(\left(1-\frac{\tau \beta}{G}\right) \cos 2 \theta+\frac{\tau(1-2 \nu)}{G}-\frac{\beta}{1-2 \nu}\right) \geq 0
\end{gathered}
$$


Since $\tau<G$, the above is equivalent to

$$
\left(\cos 2 \theta-\frac{f_{\sigma}}{1-2 \nu}\right)\left(\cos 2 \theta-\frac{\beta G-\tau(1-2 \nu)^{2}}{(1-2 \nu)(G-\tau \beta)}\right) \geq 0 .
$$

This proves part (b).

A. Examples. We now give an example, for representative values of the parameters, of the range of values for $\theta$ for which the fast plastic wave speeds exceed the fast elastic wave speeds. We compute these values for both the case where rotational terms of the Jaumann stress rate are neglected, and where they are included. The regions in stress space for which $\eta_{1}<\mu_{1}$ are determined by (25) or (30). These regions are symmetric with respect to the $x$-axis, the $y$-axis, and the origin. When the rotational terms are neglected, the region in the first quadrant is given by $\theta_{\min }<\theta<\theta_{\max }$, where $\theta_{\min }=$ $\cos ^{-1}\left(\max \left\{\beta, f_{\sigma}\right\} /(1-2 \nu)\right)$ and $\theta_{\max }=\cos ^{-1}\left(\min \left\{\beta, f_{\sigma}\right\} /(1-2 \nu)\right)$. When the rotational terms are included, (30) implies that $\tilde{\eta}_{1}<\tilde{\mu}_{1}$ when $\theta_{\min }^{J}<\theta<\theta_{\max }^{J}$, where $\theta_{\min }^{J}=$ $\cos ^{-1}\left(\max \left\{\tilde{\beta}, f_{\sigma}\right\} /(1-2 \nu)\right)$ and $\theta_{\max }^{J}=\cos ^{-1}\left(\min \left\{\tilde{\beta}, f_{\sigma}\right\} /(1-2 \nu)\right)$.

In what follows we assume that the shear modulus $G$ is $33 \mathrm{MPa}$, the Poisson's ratio $\nu$ is 0.15 and the mean shear stress is $\sigma=200 \mathrm{kPa}$. These values are used by Schaeffer in [11]. We compute the limiting values of $\theta$ for values of $f_{\sigma}$ and $\beta$ given in Table I of [11]. These values correspond to increasing shear strain $\gamma^{(t)}$, and are duplicated in the table below.

The small difference in the limiting angles for both models (with and without the rotational terms) is not surprising given that $\tilde{\beta}=\beta+O(\tau / G)$ and $\tau / G \approx 1 / 165$.

Notice that, in both cases, the sector where the reverse ordering of the waves occurs is roughly 0.5 radians wide in the first quadrant (see Figure 2), or approximately $1 / 3$ of the possible stress orientations. As the material loads, this sector includes directions for planar waves closely aligned with the direction of major principal stress. Since the major principal stress axis is aligned with the principal direction of plastic compression by (6), (7), this may be expected to be an important direction for propagation of waves. Therefore, values of the stress whose major principal direction is close to the direction of wave propagation cannot be excluded from the wave analysis.

\begin{tabular}{|c|cc||cc||cc|}
\hline$\gamma^{(t)}$ & $f_{\sigma}$ & $\beta$ & $\theta_{\min }$ & $\theta_{\max }$ & $\theta_{\min }^{J}$ & $\theta_{\max }^{J}$ \\
\hline 0.02 & 0.5873 & .1353 & 0.28767 & 0.68814 & 0.28768 & 0.68606 \\
0.03 & 0.6323 & .2044 & 0.22171 & 0.63724 & 0.22171 & 0.63521 \\
0.04 & 0.6574 & .2462 & 0.17533 & 0.60569 & 0.17533 & 0.60371 \\
0.05 & 0.6735 & .2753 & 0.13802 & 0.58329 & 0.13802 & 0.58134 \\
0.06 & 0.6847 & .2972 & 0.10473 & 0.56615 & 0.10473 & 0.56423 \\
0.07 & 0.6929 & .3144 & 0.07127 & 0.55249 & 0.07127 & 0.55059 \\
\hline
\end{tabular}




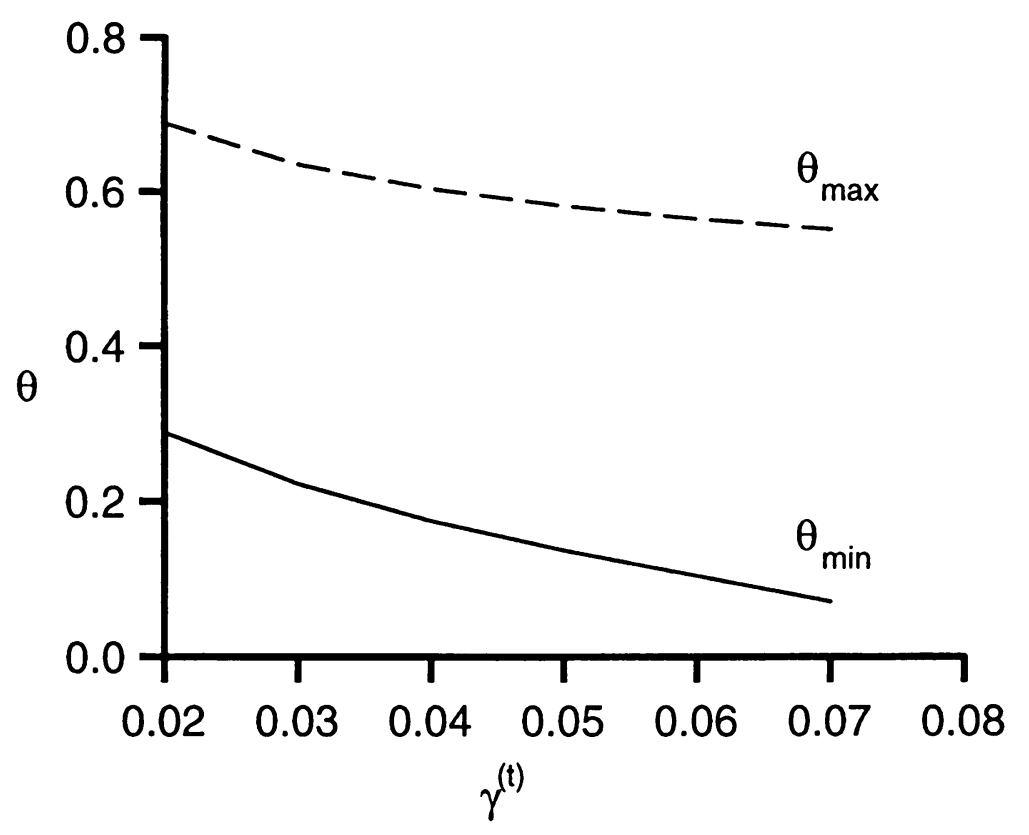

FIG. 2. $\theta_{\min }$ and $\theta_{\max }$ during loading

\section{REFERENCES}

[1] L. An, Loss of hyperbolicity in elastic-plastic material at finite strains, SIAM J. Appl. Math. 53, 621-654 (1993)

[2] L. An and D. G. Schaeffer, The flutter instability in granular flow, J. Mech. Physics Solids 40, 683-698 (1992)

[3] D. C. Drucker, R. E. Gibson, and D. J. Henkel, Soil mechanics and work-hardening theories of plasticity, Trans. American Soc. Civil Engrg. 122, 338-346 (1957)

[4] P. V. Lade and J. M. Duncan, Elastoplastic stress-strain theory for cohesionless soil, J. Geotech. Engrg. 101, 1037-1053 (1975)

[5] P. LeFloch, G. Dal Maso, and F. Murat, Definition of weak stability of nonconservative products, Preprint, Ecole Polytechnique, France, 1989

[6] P. LeFloch and T. P. Liu, Existence theory for nonlinear hyperbolic systems in nonconservative form, Forum Mathematicum, 1992 (to appear)

[7] J. Mandel, Conditions de stabilité et postulate de Drucker, Rheology and Soil Mechanics, IUTAM Symposium at Grenoble (G. Kravtchenko and P. Sirieys, eds.), 58--68 (1964)

[8] H. B. Poorooshasb, I. Holubec, and A. N. Sherbourne, Yielding and flow of sand in triaxial compression: Parts II and III, Canadian Geotech. J. 4, 376-397 (1976)

[9] J. Rice, The localization of plastic deformation, Proc. IUTAM Congress at Delft (W. Koiter, ed.), 207-220 (1976)

[10] I. Sandler and D. Rubin, The consequences of non-associated plasticity in dynamic problems, Constitutive Laws for Engineering Materials: Theory and Applications (C. S. Desai et al., ed.), Elsevier, New York, 1987, pp. 345-352

[11] D. G. Schaeffer, Instability and ill-posedness in the deformation of granular materials, International Journal for Numerical Analysis Methods in Geomechanics 14, 253-278 (1990) 
[12] D. G. Schaeffer and Michael Shearer, Scale-invariant initial value problems in one-dimensional dynamic elastoplasticity, with consequences for multidimensional nonassociative plasticity, European Journal of Applied Mathematics 3, 225-254 (1992)

[13] V. V. Sokolovskii, D. H. Jones, and A. N. Schofield, Statics of Soil Media, Butterworths Scientific Publications, London, 1960

[14] I. Vardoulakis and B. Graf, Calibration of constitutive models for granular materials using data from experiments, Géotechnique 35, 299-317 (1985) 